Open Access

\title{
Microcirculatory perfusion shows wide inter-individual variation and is important in determining shock reversal during resuscitation in a porcine experimental model of complex traumatic hemorrhagic shock
}

Sam D. Hutchings ${ }^{1,2,3^{*}}$, David N. Naumann ${ }^{1,4}$, Sarah Watts ${ }^{3}$, Callie Wilson ${ }^{3}$, Clare Burton ${ }^{3}$, Julia Wendon ${ }^{2}$ and Emrys Kirkman ${ }^{3}$

\footnotetext{
* Correspondence: sam.hutchings@ kcl.ac.uk

${ }^{1}$ Royal Centre for Defence Medicine, Birmingham Research Park, Vincent Drive, Birmingham B15 2SQ, UK ${ }^{2}$ Department of Critical Care, King's College Hospital London, Denmark Hill, London SE5 9RS, UK Full list of author information is available at the end of the article
}

\begin{abstract}
Background: Traumatic hemorrhagic shock (THS) is a leading cause of preventable death following severe traumatic injury. Resuscitation of THS is typically targeted at blood pressure, but the effects of such a strategy on systemic and microcirculatory flow remains unclear. Failure to restore microcirculatory perfusion has been shown to lead to poor outcomes in experimental and clinical studies. Systemic and microcirculatory variables were examined in a porcine model of complex THS, in order to investigate inter-individual variations in flow and the effect of microcirculatory perfusion on reversal of the shock state.

Methods: Baseline standard microcirculatory variables were obtained for 22 large white pigs using sublingual incident dark field (IDF) video-microscopy. All animals were subjected to a standardised hind-limb injury followed by a controlled haemorrhage of approximately $35 \%$ of blood volume (shock phase). This was followed by 60 min of fluid resuscitation with either $0.9 \%$ saline or component blood products and a target $\mathrm{SBP}$ of $80 \mathrm{mmHg}$ (early resuscitation phase). All animals were then given blood products to a target SBP of $110 \mathrm{mmHg}$ for $120 \mathrm{~min}$ (mid-resuscitation phase), and a further 100 min (late resuscitation phase). IDF readings were obtained at the midpoint of each of these phases. Cardiac output was measured using a pulmonary artery catheter. Animals were divided into above average (A) and below average (B) perfused vessel density (PVD) groups based on the lowest recorded PVD measurement taken during the shock and early resuscitation phases.

(Continued on next page)
\end{abstract}


(Continued from previous page)

Results: There was minimal inter-individual variation in blood pressure but wide variation of both systemic and microcirculatory flow variables during resuscitation. During shock and early resuscitation, group A $(n=10)$ had a mean PVD of 10.5 $(\mathrm{SD} \pm 2.5) \mathrm{mm} / \mathrm{mm}^{2}$ and group $B(n=12) 5.5(\mathrm{SD} \pm 4.1) \mathrm{mm} / \mathrm{mm}^{2}$. During the later resuscitation phases, group A maintained a significantly higher PVD than group B. Group A initially had a higher cardiac output, but the difference between the groups narrowed as resuscitation progressed. At the end of resuscitation, group A had significantly lower plasma lactate, higher lactate clearance, lower standard base deficit and smaller mixed venous-arterial $\mathrm{CO}_{2}$ gradient. There was no significant difference in blood pressure between the two groups at any stage.

Conclusion: There was a wide variation in both macro- and microcirculatory flow variables in this pressure-targeted experimental model of THS resuscitation. Early changes in microvascular perfusion appear to be key determinants in the reversal of the shock state during resuscitation. Microcirculatory flow parameters may be more reliable markers of physiological insult than pressure-based parameters and are potential targets for goal-directed resuscitation.

Keywords: Haemorrhagic shock, Microcirculation, Traumatic injury, Sublingual video-microscopy, Lactate clearance, $\mathrm{CO}_{2}$ gap, Blood products, Perfused vessel density

\section{Background}

Traumatic hemorrhagic shock (THS) is a leading preventable cause of death following severe traumatic injury [1-3], and timely reversal of the shock state is essential in reducing post-injury morbidity and mortality.

Resuscitation from THS is invariably conducted with reference to a pressure-based paradigm that currently emphasises so-called "permissive hypotension". This strategy is based on the hypothesis that excessive blood and fluid administration, given with the goal of achieving normotension, may lead to clot disruption and increased bleeding. This is seemingly well supported by experimental [4] and clinical [5] evidence and recommended by expert authorities [6, 7]. Crucially, however, the effects of pressuretargeted resuscitation upon blood flow, especially microcirculatory blood flow, are less well defined.

The microcirculation is a collective term for the network of small blood vessels that perform the final role in the delivery of cellular oxygen and substrate and is therefore of paramount importance during shock and resuscitation. Small animal studies have shown that the ability to maintain microcirculatory perfusion is critical to outcome following THS [8]. However, these studies utilised models of simple haemorrhage and may have limited translation into clinical practice. To date, only one published clinical study has examined the effects of microcirculatory impairment on end-organ function following THS [9], finding that there was an association between microcirculatory impairment and organ failure. However, this study examined the microcirculation in the period after haemodynamic stability had been restored, not during the shock phase itself.

If it could be demonstrated that individuals with THS who were able to maintain higher systemic and microcirculatory blood flows during resuscitation had improved outcomes, then this could potentially identify avenues for the development of novel end points of resuscitation based on flow-based parameters. 
The current study presents data on macro- and microcirculatory blood flow collected from a porcine experimental model of THS. The aims of the study are to investigate (i) inter-individual variations in systemic and microcirculatory blood flow, (ii) the relationship between systemic and microcirculatory blood flow and (iii) to examine whether animals which maintained a higher degree of microcirculatory perfusion during shock and resuscitation had more effective and timely reversal of the shock state.

\section{Methods}

\section{Study design}

This is an opportunistic prospective observational study that investigates the haemodynamic and microcirculatory variables for some of the subjects of a previously published porcine experimental study. The original experimental study was undertaken to address the effects of different initial resuscitation fluids on the development of trauma-induced coagulopathy, the results of which have been published elsewhere [10]. Data were obtained from experiments conducted with the presence of an investigator trained in the acquisition of microcirculatory images.

\section{Animal preparation}

The whole experiment was approved and conducted under license from the Home Office Animals (Scientific Procedures) Act 1986.

The full animal preparation is described in detail elsewhere [10]. In summary, female large white pigs $(43-56 \mathrm{~kg})$ were terminally anaesthetised and allowed spontaneous respiration following tracheal intubation. The left femoral artery and left common carotid arteries were cannulated to allow the withdrawal of blood and infusion of resuscitative fluid, respectively. Cardiac output monitoring was performed using a pulmonary artery catheter (Vigilance Volumetrics CEDV; Edwards Lifesciences Ltd). The bladder was catheterised. A midline laparotomy and splenectomy was performed, and the abdomen was closed.

\section{Experimental protocol}

The full experimental protocol is detailed previously [10, 11] and was designed to replicate battlefield injury, shock and subsequent resuscitation. The experiment was designed to keep all animals alive until the end of resuscitation. A proportion of animals received a controlled blast injury, and all animals received a controlled hindlimb injury from a captive bolt gun as described elsewhere [10]. Following the haemorrhagic shock phase, animals were assigned to receive initial resuscitation with either (a) $0.9 \%$ saline or (b) component blood therapy consisting of leucodepleted porcine red blood cells and plasma (delivered in a 1:1 ratio). The sampling, storage and preparation of these blood products are described in detail elsewhere [10].

Data was collected at five time points as follows:
(a) Baseline (pre-injury)
(b)Shock (30 min after controlled haemorrhage and injury)
(c) Early (hypotensive) resuscitation (30 min after commencement of resuscitation)
(d)Mid-resuscitation (90 min after commencement of resuscitation) 
(e) Late resuscitation (170 min after commencement of resuscitation)

In accordance with currently accepted trauma shock resuscitation protocols, the target systolic blood pressure was set at $80 \mathrm{mmHg}$ for the early resuscitation phase and $110 \mathrm{mmHg}$ during subsequent resuscitation phases. Animals were given boluses of fluid to achieve these pressure targets. During early resuscitation, animals were treated with either 0.9 \% saline or blood products. During mid- and late resuscitation, all animals exclusively received blood products.

\section{Microcirculatory monitoring}

The microcirculation was assessed using incident dark field (IDF) video-microscopy (Cytocam, Braedius Medical B.V), which has been recently validated against a precursor device in this setting [12]. Video clips of the microcirculation were acquired from the sublingual area at each of the five time points. Images were acquired by three operators but reviewed for quality by a single experienced operator. At least three (and ideally five) video sequences, of at least five seconds duration, were recorded at each time point, in accordance with the current consensus for assessing the microcirculation [13]. Quality assessment criteria were used to optimise image quality according to standard practice [14]. Each video sequence was assigned a five-digit random number when saved to digital media. Images were therefore de-identified with respect to animal, treatment and time point.

\section{Analysis of microcirculation video clips}

Analysis was conducted offline by a single operator (S.H.) using semi-automated software (Automated Vascular Analysis v. 3.0, Microvision Medical, NL). All vessels were identified and traced by hand. Only small vessels $(<20 \mu \mathrm{m}$ diameter $)$ were included in the analysis. Results were obtained for the following variables:

(i) Total vessel density (TVD); the total numbers of vessels in the visual field.

(ii) Microcirculatory flow index (MFI), originally described by Boerma et al. (27) rates each quadrant of the image in terms of the most appropriate flow rating $(0=$ no flow; 1 = intermittent flow; 2 = sluggish flow; 3 = continuous flow). To improve the accuracy of this measurement, we ascribed MFI values to each individual vessel segment and the mean value for each video sequence was calculated.

(iii) Perfused vessel density (PVD); the total number of vessels in the visual field with either sluggish or continuous flow.

\section{Microcirculatory perfusion}

Animals were divided ex priori into two groups classed as having above average (group A) and below average (group B) microcirculatory perfusion based on their lowest PVD values. PVD was selected as the discriminator as it is a composite marker composed of both vessel density and flow data. Firstly, the lowest PVD during the shock and early resuscitation phases was calculated for each of the 22 animals. Then the mean of all these PVD values (for all animals) was selected as the reference point above and below which groups A and B were, respectively, divided. 


\section{End points of resuscitation}

Since the experimental protocol was designed for all animals to survive, we chose endpoints reflective of the degree of shock reversal. The primary measure of shock reversal was lactate clearance (\% change between early and late resuscitation time points). Lactate concentration, standard base excess and the gradient between mixed venous and arterial $\mathrm{PCO}_{2}\left(\mathrm{CO}_{2}\right.$ gap $)$ at the late resuscitation time point were selected as secondary endpoints. Urine output was used as a surrogate marker of renal perfusion.

\section{Statistical analysis}

Data analysis was performed using Prism version 6.0 (GraphPad). Data was assessed for normality using D’Agostino-Pearson omnibus normality test. Repeated $t$ tests or Mann-Whitney $U$ tests with Holm-Sidak correction for multiple comparisons were used to assess for differences between groups. A $p$ value of $<0.05$ was considered significant.

\section{Results}

Data was collected on twenty-two animals which survived to the end of resuscitation. During initial resuscitation, 12 animals received blood products and ten $0.9 \%$ saline. Ten animals were exposed to blast.

\section{Inter-individual variation in macro- and micro-haemodynamic variables}

Macro-circulatory flow and pressure variables and microcirculatory perfusion variables showed a similar trend during shock and resuscitation (Fig. 1a-c). However, although blood pressure, as the targeted parameter, showed little variation between animals (Fig. 1a), there was a wider variation in both cardiac output (Fig. 1b) and PVD (Fig. 1c).

During the shock and early resuscitation phases, the mean of the lowest recorded PVD for all animals was $6.2 \mathrm{~mm} / \mathrm{mm}^{2}$ and this value was used to divide animals into groups with above average (group A) and below average (group B) microcirculatory perfusion. Illustrative IDF video sequences from these two groups are provided in the supplementary electronic information (Additional file 1: Video 1 and Additional file 2: Video 2).

Expectedly, there was no difference in blood pressure between animals in groups A and B (Fig. 2a). PVD in animals with above average perfusion during shock and early resuscitation returned to baseline by the end of the experiment in contrast to those in the below average perfusion group in whom it remained depressed (Fig. 2b). The cardiac output fell sharply following haemorrhage in both groups, but the difference between the groups was less pronounced and became non-significant as resuscitation progressed (Fig. 2c). Animals in group A had significantly lower systemic vascular resistance during the shock and early resuscitation phases than those in group B (group A $1086 \pm 296$, group B $1503 \pm 319$ dyn s $\mathrm{cm}^{-5} p=0.002$ ). By the end of the experiment, there was no difference in cardiac output between the two groups but a persisting gap in microcirculatory perfusion.

\section{Effect of microcirculatory perfusion on shock reversal}

Data on shock reversal variables are presented in Table 1. Group A had a significantly higher lactate clearance than group B. At the end of the experiment, group A also had 


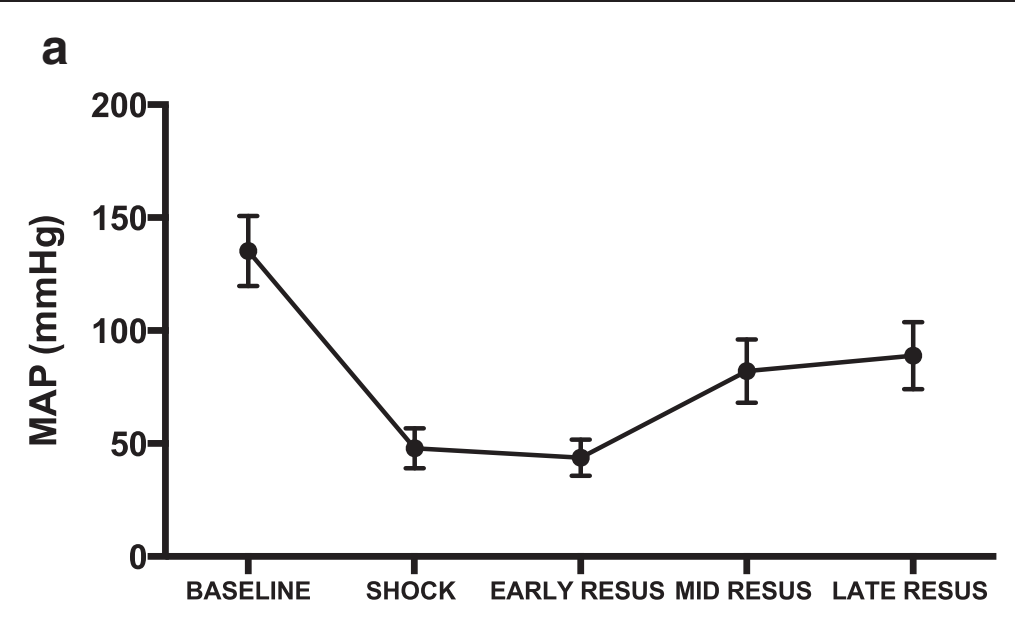

b

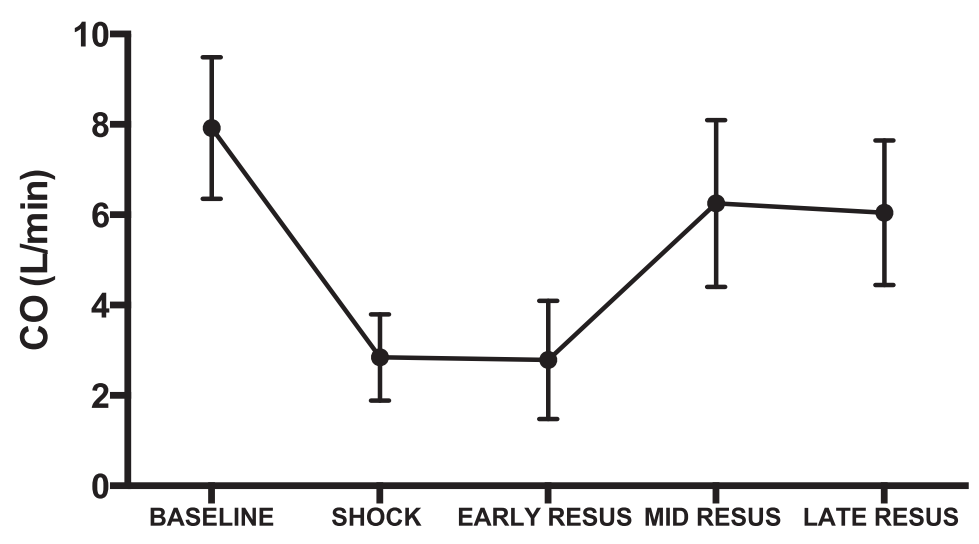

C

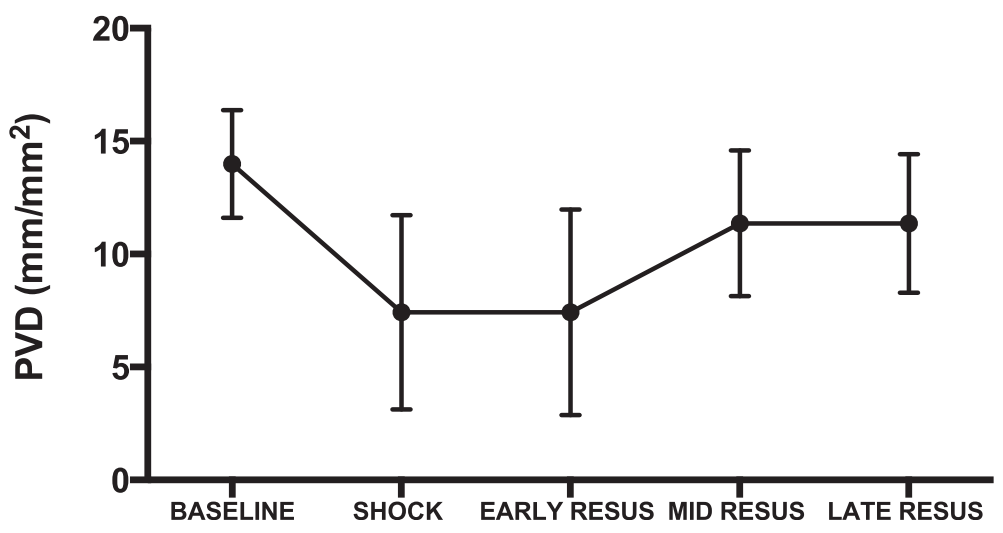

Fig. 1 Changes in mean arterial pressure (MAP) (a), cardiac output (CO) (b) and perfused vessel density (PVD) (c), during shock and resuscitation. Values shown as mean (SD) of all animals at each time point

a lower standard base deficit, lower final lactate concentration and a reduced $\mathrm{CO}_{2}$ gap compared to group B. There was a trend to higher urine output in group A, but this did not reach significance.

We further analysed each group of animals based on the type of initial resuscitation fluid and the state of microcirculatory perfusion. Although the numbers in each of 


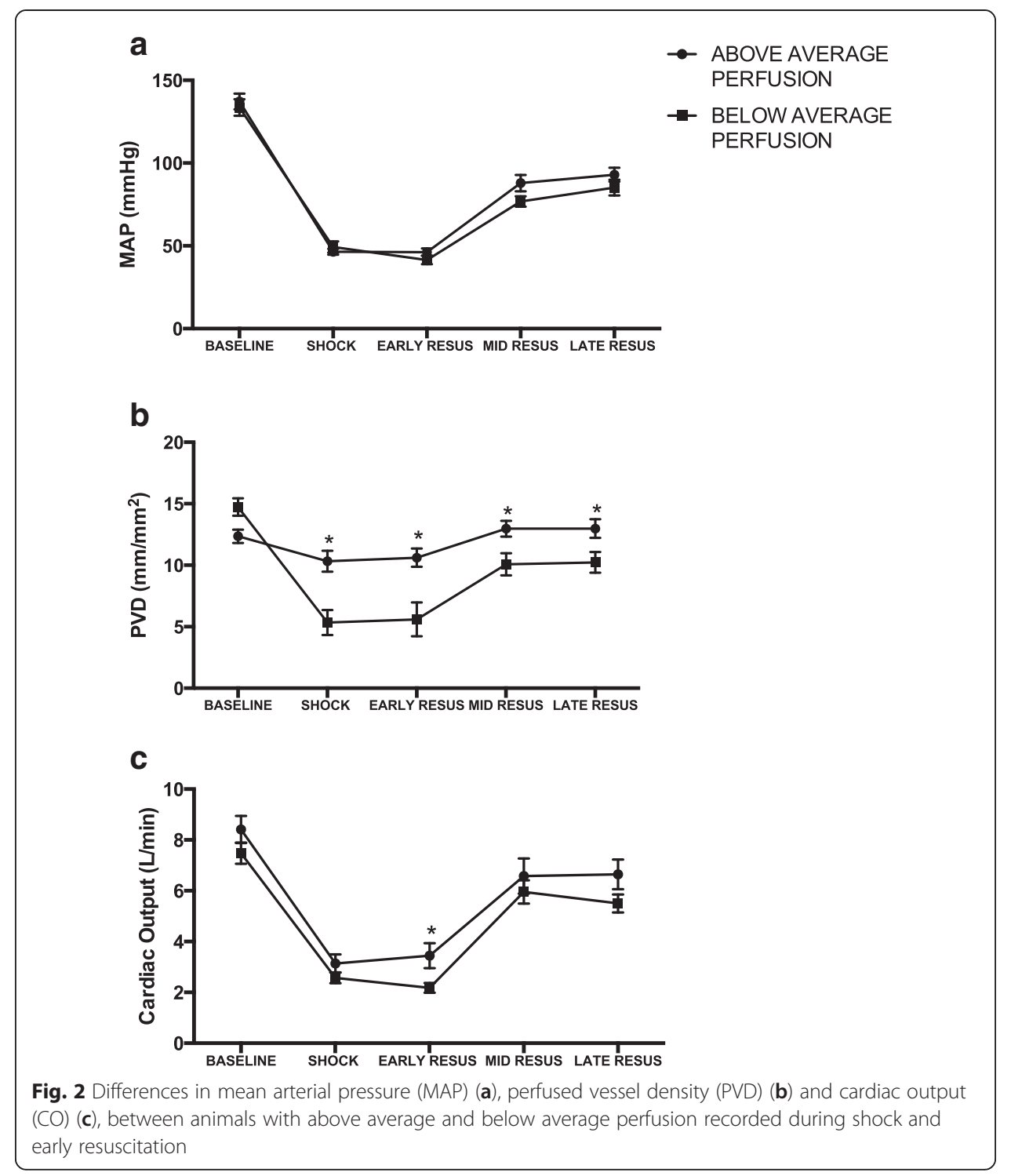

Table 1 Markers of shock reversal at the end of resuscitation in animals with above and below average perfused vessel density (PVD) recorded during shock and early resuscitation

\begin{tabular}{llll}
\hline & $\begin{array}{l}\text { Group A } \\
\text { (Above average PVD) } \\
n=10\end{array}$ & $\begin{array}{l}\text { Group B } \\
\text { (Below average PVD) } \\
n=12\end{array}$ & $p$ value \\
\hline Tissue perfusion variables, mean (SD) & & & \\
Lactate at late resuscitation (mmol/l) & $4.9(2.5)$ & $8.6(3.7)$ & $0.02^{*}$ \\
Lactate clearance during resuscitation (\%) & $73.1(11.9)$ & $40.7(32.3)$ & $<0.001^{*}$ \\
Standard base deficit at late resuscitation & $1.3(4.3)$ & $-4.8(5.7)$ & $0.01^{*}$ \\
$\left(\mathrm{PvCO}_{2}-\mathrm{PaCO}\right)$ at late resuscitation (kPa) & $1.3(0.6)$ & $2.1(0.7)$ & $0.03^{*}$ \\
Urine output $(\mathrm{ml})^{\mathrm{a}}$ & $260(163)$ & $168(152)$ & 0.25 \\
\hline
\end{tabular}

*Significant using two-tailed $t$ test

${ }^{a}$ Cumulative between shock phase and end of experiment 
these four groups was too small for statistical significance, the impact of impaired microcirculatory perfusion can be observed within each group of identically treated animals (Fig. 3).

\section{Effect of initial resuscitation fluid and blast exposure}

Animals treated with $0.9 \%$ saline as the initial resuscitation fluid had significantly lower PVD prior to treatment than those that received blood products. Although there was a suggestion that animals initially treated with $0.9 \%$ saline continued to have a lower PVD throughout subsequent resuscitation (Fig. 4), further meaningful analysis was precluded by this lack of homogeneity prior to treatment. Examining the effects of administration of initial resuscitation fluid on PVD in each individual animal also failed to reveal a uniform trend (Fig. 5). There were no discernable differences in lactate clearance or final lactate concentration based purely on the type of initial resuscitation fluid used (Additional file 3).

Ten out of twenty-two animals were exposed to blast injury. There were no apparent differences in either macro- or microcirculatory variables between animals attributable to blast exposure, nor in the lactate profile, although further analysis was confounded by the heterogeneity of fluid treatments applied (Additional file 4).

\section{Discussion}

The main finding of this study is that in a large animal model of complex traumatic injury and haemorrhagic shock, with resuscitation targeted at systolic blood pressure, there is wide inter-individual variations in both macro- and microcirculatory flow variables. Furthermore, animals which were able to maintain flow, both at the systemic and microcirculatory level, had more timely reversal of the shock state during resuscitation.

Our study adds to the previous work conducted by Dubin and co-workers [15] who showed that microcirculatory flow fell in line with cardiac output during the early phases of haemorrhage in an ovine model. Our study supports these findings but further shows that there is a group of animals in whom the reduction in microcirculatory perfusion appears less marked and that these animals exhibit a more rapid reversal

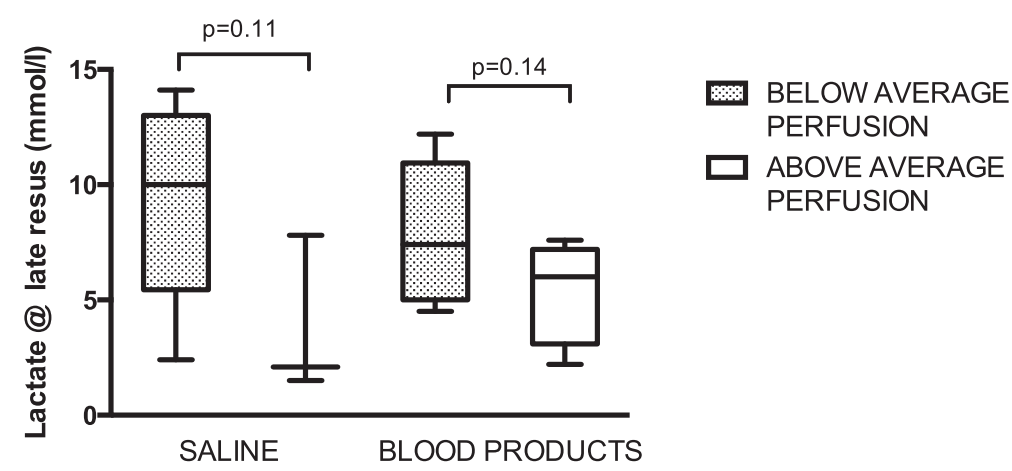

Fig. 3 Differences in lactate concentration at the end of resuscitation between animals with above and below average microcirculatory perfusion recorded during shock and early resuscitation. Animals are grouped by initial resuscitation fluid 


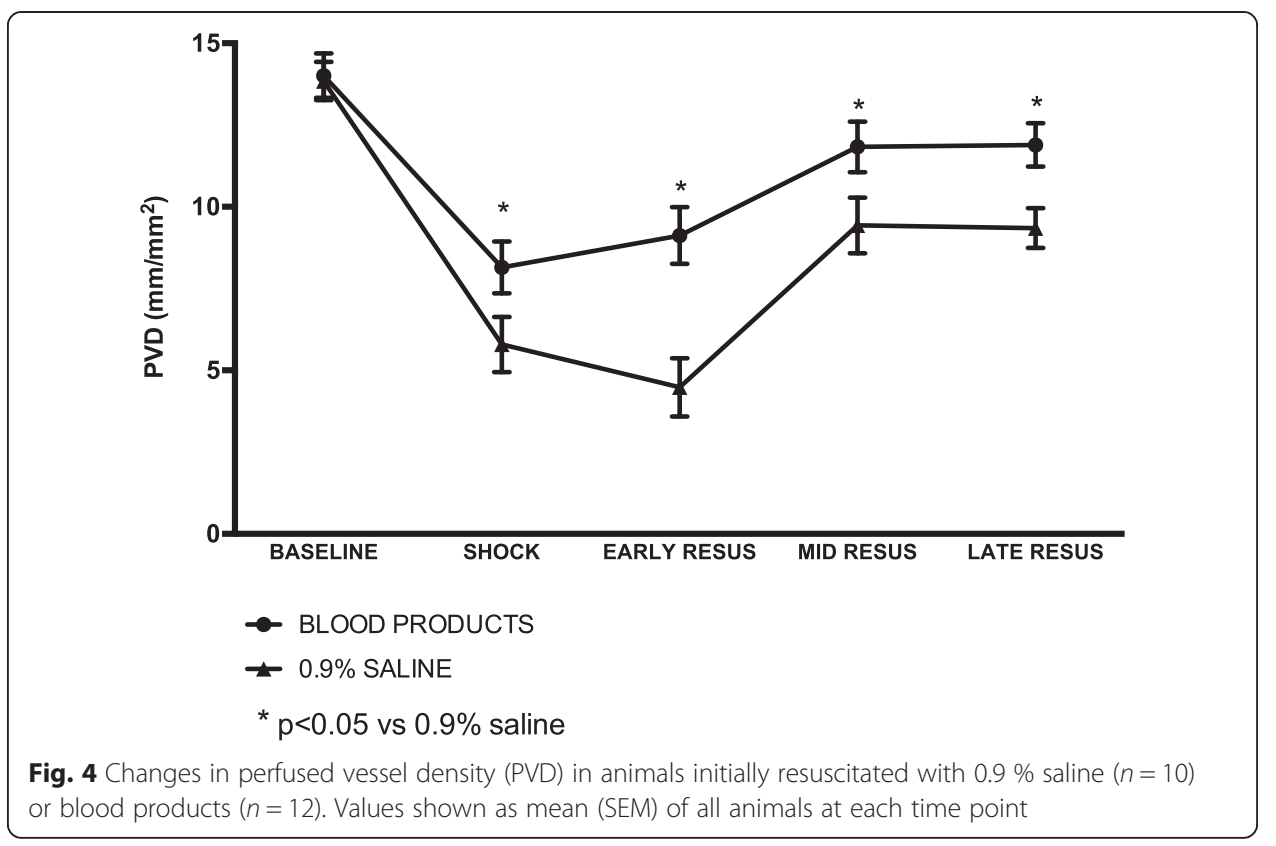

of the shock state during subsequent resuscitation. Although microcirculatory flow did initially follow cardiac output in our experimental model, the coherence between macro- and microcirculatory flow was, to some extent, lost during late resuscitation.

A feasible physiological explanation of our study findings is that those animals with below average microcirculatory perfusion during shock and early resuscitation have responded to a fall in cardiac output with excessive vasoconstriction compared to animals with above average perfusion. Such excessive vasoconstriction, above that required to maintain organ perfusion pressure, may lead to microcirculatory impairment and consequent tissue hypo-perfusion, leading to the reduced lactate clearance observed in this group. Precisely why some animals responded to an equivalent insult with differing degrees of vasoconstriction remains unexplained, and further investigative work is required to elucidate the exact mechanistic processes involved.

In the clinical setting, the identification of patients who have poor microcirculatory flow during shock and early resuscitation may potentially lead to important changes in management. Such an approach could see the introduction of more individualised therapy, and this would have a better physiological rationale than a blanket 'one size fits all' pressure-targeted resuscitation strategy. $\mathrm{Xu}$ and co-workers elegantly demonstrated that targeting microcirculatory perfusion rather than blood pressure leads to less fluid administration with no detriment to outcome in a porcine model of THS [16]. The use of hand-held video microscopes combined with a point-of-care assessment tool for bedside video analysis may allow the introduction of such a strategy into clinical practice. There are early indications that this may be feasible [17]. Adoption of such systems into clinical practice may allow the development of new resuscitation protocols for THS based around flow rather than pressure targets.

Failure to normalise the microcirculation following resuscitation has significant effects on organ function [18]. It is common for critically ill septic patients to have a disjunction between macro- and microcirculatory function, and this has also been 


\section{BLOOD PRODUCTS}

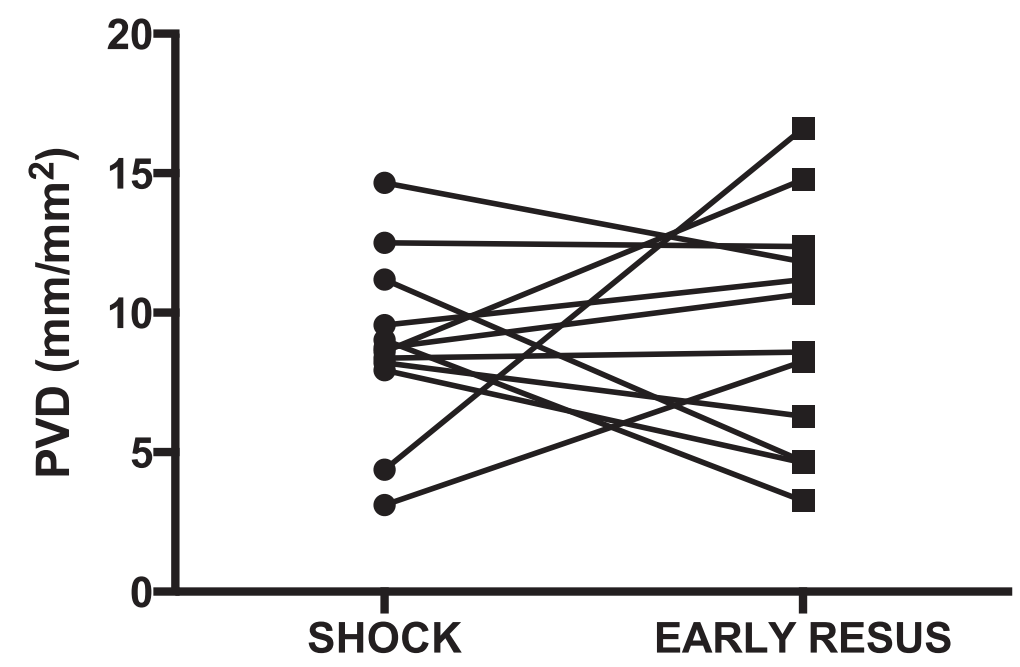

$0.9 \%$ SALINE

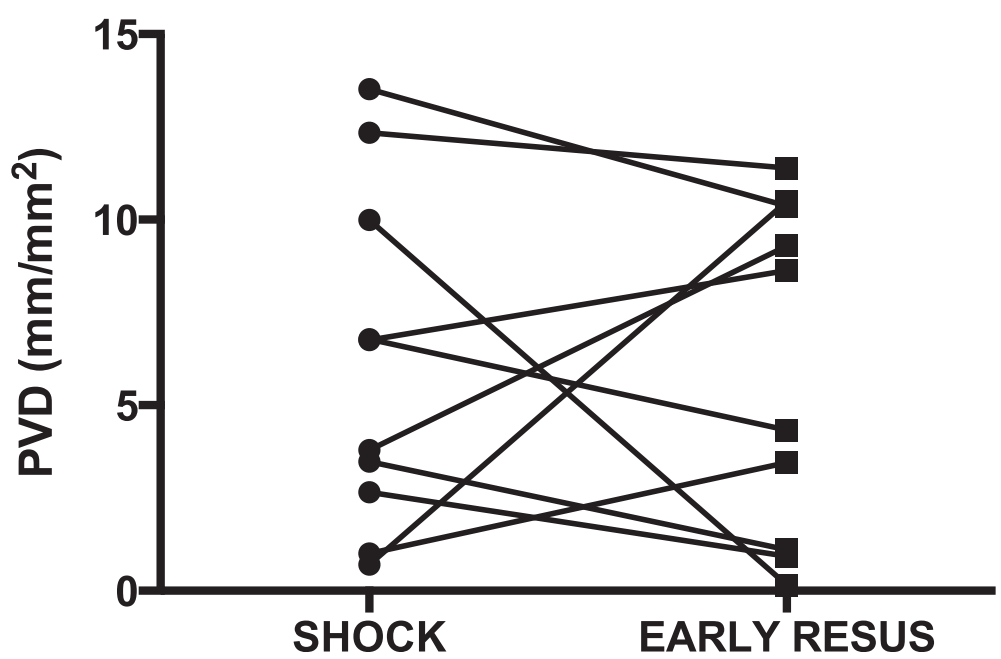

Fig. $\mathbf{5}$ Changes in perfused vessel density (PVD) in individual animals after administration of initial resuscitation fluid. Values are recorded $15 \mathrm{~min}$ before (SHOCK) and $30 \mathrm{~min}$ after (EARLY RESUS) commencement of fluid resuscitation

recently demonstrated in patients following THS [9]. Previous preclinical studies of experimental haemorrhagic shock have variably demonstrated either recovery [19] or persistence $[20,21]$ of microcirculatory dysfunction after systemic haemodynamic variables have been restored. Whilst our results show that microcirculatory perfusion broadly follows systemic blood flow in returning to normal levels following resuscitation, there were a group of animals in whom the microcirculation continued to be disproportionately impaired. Our results suggest that those animals which show very significant falls in microcirculatory impairment during the shock period never return to baseline values of perfusion, in contrast to those with more modest initial falls in 
perfused vessel density. Possible explanations for persisting microcirculatory failure after restoration of systemic blood flow include dysfunction of any or all of the individual elements that make up the microcirculation, for example, endothelial cell swelling caused by ischaemia and re-perfusion injury [22], clogging of small vessels with activated leucocytes [23] and dysfunction of transfused stored erythrocytes leading to vessel occlusion [24]. Our study was only designed to assess the initial response of the microcirculation to shock and resuscitation, and further studies are required to answer these interesting questions relating to the precise mechanisms behind persistent microcirculatory dysfunction.

Our study was opportunistic in nature and although we prospectively collected data, we not unsurprisingly ended up with groups of animals which had received a variety of heterogeneous injuries and treatments. We did examine the data to determine if there was any discernible effect of differing fluid administration on microcirculatory perfusion in animals which received saline and blood products but failed to find a unifying pattern. In part, this was because animals which received saline had a significantly lower PVD during the shock phase prior to initial treatment, precluding subsequent analysis. Other studies have appeared to show enhanced microcirculatory perfusion following the administration of fluids with higher viscosities [25, 26], but many of these were conducted in small animals with limited translatability into the clinical setting. Furthermore, of the small number of large animal studies conducted in this field [27, 28], none examined complex injury as opposed to simple blood loss, again limiting the potential for translation. The interesting question of which fluid more effectively resuscitates the microcirculation is still not fully answered, and further studies specifically designed to address this question in a model of complex THS would be of value.

Our study has several limitations, the most obvious being the heterogeneity of the applied treatments. In order to reduce the use of animals, we collected data from an existing experimental model, whose primary aim was to detect differences in trauma coagulopathy based on the choice of initial resuscitation fluid. Furthermore, in order to replicate recent military combat conditions, some animals were exposed to blast injury. We were unable to control for these variations in our small sample size.

Despite the pre-allocation of animals into $0.9 \%$ saline and blood product groups, these groups were statistically different, in terms of PVD, even before the start of resuscitation. This made further detailed comparison of animals based on the type of fluid difficult.

The authors also recognise the limitation of not having survival as an outcome measure in this observational study; ideally, this would be a useful translatable outcome for clinical relevance. However, the experiment was designed for all animals to survive until the end of the protocol. Therefore, surrogate markers of shock reversal such as lactate clearance, base excess, and the " $\mathrm{CO}_{2}$ gap" [29] were used as measures of resuscitative 'success'.

\section{Conclusions}

In a large animal model of complex hemorrhagic shock and pressure-targeted resuscitation, there was a wide variation in both macrocirculatory flow and microcirculatory perfusion. Pressure-based monitoring did not discriminate between animals with 
poor and preserved perfusion. Animals with above average microcirculatory perfusion had more timely reversal of the shock state during subsequent resuscitation. If translated to the clinical setting, microcirculatory perfusion may represent a useful endpoint of resuscitation and a potential therapeutic target.

\section{Approvals}

The whole experiment was approved and conducted under license from the UK Home Office Animals (Scientific Procedures) Act 1986.

\section{Additional files}

Additional file 1: Video 1. IDF video sequence taken from animal with above average microcirculatory perfusion during early resuscitation phase. (MP4 $8730 \mathrm{~kb}$ )

Additional file 2: Video 2. IDF video sequence taken from animal with below average microcirculatory perfusion during early resuscitation phase. (MP4 18187 kb)

Additional file 3: Further information relating to the effects of initial resuscitation fluid on systemic haemodynamic and microcirculatory variables. (PDF $39 \mathrm{~kb}$ )

Additional file 4: Effect of blast injury on systemic haemodynamic and microcirculatory variables. (PDF 35 kb)

\section{Abbreviations}

CO, cardiac output; IDF, incident dark field; IQR, inter-quartile range; MAP, mean arterial pressure; MFI, microcirculatory flow index; PAC, pulmonary artery catheter; $\mathrm{PaCO}$, arterial carbon dioxide tension; $\mathrm{PvCO}$, mixed venous carbon dioxide tension; PVD, perfused vessel density; SBP, systolic blood pressure; SBE, standard base excess; SEM, standard error of the mean; SD, standard deviation; SVR, systemic vascular resistance; TVD, total vessel density

\section{Competing interests}

The authors declare that they have no competing interests. All authors except JW are employed by the UK Ministry of Defence.

\section{Authors' contributions}

$\mathrm{SH}$ conceptualised the study, assisted with design of the protocol, collected and analysed data and wrote the first draft and revision of the manuscript. DN revised the manuscript and assisted with the literature review. JW advised on the concept and design of the study and critically appraised the manuscript. CB and CW collected the data and critically reviewed the manuscript. SW and EK designed the protocol, collected the data and critically appraised the manuscript. All authors read and approved the final manuscript.

\section{Funding}

This study was funded by the UK Ministry of Defence.

\section{Author details}

${ }^{1}$ Royal Centre for Defence Medicine, Birmingham Research Park, Vincent Drive, Birmingham B15 2SQ, UK. ${ }^{2}$ Department of Critical Care, King's College Hospital London, Denmark Hill, London SE5 9RS, UK. ${ }^{3}$ Defence Science and Technology Laboratory, Porton Down, Salisbury, Wiltshire SP4 0JQ, UK. ${ }^{4}$ NIHR Surgical Reconstruction and Microbiology Research Centre, Queen Elizabeth Hospital, Birmingham B152TH, UK.

Received: 9 February 2016 Accepted: 30 May 2016

Published online: 24 June 2016

\section{References}

1. Geeraedts LMG, Kaasjager HAH, van Vugt AB, Frölke JPM (2009) Exsanguination in trauma: a review of diagnostics and treatment options. Injury 40:11-20. doi:10.1016/j.injury.2008.10.007

2. Curry N, Hopewell S, Doree C et al (2011) The acute management of trauma hemorrhage: a systematic review of randomized controlled trials. Crit Care 15:R92. doi:10.1186/cc10096

3. Kauvar DS, Lefering R, Wade CE (2006) Impact of hemorrhage on trauma outcome: an overview of epidemiology, clinical presentations, and therapeutic considerations. J Trauma 60:S3-11. doi:10.1097/01.ta.0000199961.02677.19

4. Bai X, Yu W, Ji W et al (2015) Resuscitation strategies with different arterial pressure targets after surgical management of traumatic shock. Crit Care 19:1498-22. doi:10.1186/s13054-015-0897-6

5. Bickell WH, Wall MJ Jr, Pepe PE et al (1994) Immediate versus delayed fluid resuscitation for hypotensive patients with penetrating torso injuries. N Engl J Med 331:1105-1109. doi:10.1056/NEJM199410273311701

6. National Institute for Clinical Excellence (NICE) (2004) Pre-hospital initiation of fluid replacement therapy in trauma. nice.org.uk/guidance/ta74

7. Spahn DR, Bouillon B, Cerny V et al (2013) Management of bleeding and coagulopathy following major trauma: an updated European guideline. Crit Care 17:R76. doi:10.1186/cc12685

8. Kerger H, Waschke KF, Ackern KV et al (1999) Systemic and microcirculatory effects of autologous whole blood resuscitation in severe hemorrhagic shock. Am J Physiol 276:H2035-43 
9. Tachon G, Harrois A, Tanaka S et al (2014) Microcirculatory alterations in traumatic hemorrhagic shock*. Crit Care Med 42:1433-1441. doi:10.1097/CCM.0000000000000223

10. Watts S, Nordmann G, Brohi K et al (2015) Evaluation of prehospital blood products to attenuate acute coagulopathy of trauma in a model of severe injury and shock in anesthetized pigs. Shock 44(Suppl 1):138-148. doi:10.1097/SHK.0000000000000409

11. Garner JP, Watts S, Parry C et al (2009) Development of a large animal model for investigating resuscitation after blast and hemorrhage. World J Surg 33:2194-2202. doi:10.1007/s00268-009-0105-4

12. Hutchings S, Watts S, Kirkman E (2015) The Cytocam video microscope. A new method for visualising the microcirculation using Incident Dark Field technology. Clin Hemorheol Microcirc 62:261-271. doi:10.3233/CH-152013

13. De Backer D, Hollenberg S, Boerma C et al (2007) How to evaluate the microcirculation: report of a round table conference. Crit Care 11(5):R101

14. Massey MJ, Larochelle E, Najarro G et al (2013) The microcirculation image quality score: development and preliminary evaluation of a proposed approach to grading quality of image acquisition for bedside videomicroscopy. J Crit Care 28:913-917. doi:10.1016/j.jcrc.2013.06.015

15. Dubin A, Pozo MO, Ferrara G et al (2009) Systemic and microcirculatory responses to progressive hemorrhage. Intensive Care Med 35:556-564. doi:10.1007/s00134-008-1385-0

16. Xu JJ, Ma LL, Sun SS et al (2013) Fluid resuscitation guided by sublingual partial pressure of carbon dioxide during hemorrhagic shock in a porcine model. Shock 39:361-365. doi:10.1097/SHK.0b013e31828936aa

17. Lima A, López A, van Genderen ME et al (2015) Interrater reliability and diagnostic performance of subjective evaluation of sublingual microcirculation images by physicians and nurses. Shock 44:239-244. doi:10.1097/SHK.0000000000000401

18. Ince C (2005) The microcirculation is the motor of sepsis. Crit Care 9(Suppl 4):S13-9. doi:10.1186/cc3753

19. Cheung AT, Duong PL, Driessen B et al (2006) Systemic function, oxygenation and microvascular correlation during treatment of hemorrhagic shock with blood substitutes. Clin Hemorheol Microcirc 34:325-334

20. Paes-da-Silva F, Gonzalez AP, Tibiriçá E (2003) Effects of fluid resuscitation on mesenteric microvascular blood flow and lymphatic activity after severe hemorrhagic shock in rats. Shock 19:55-60

21. Cabrales $P$, Intaglietta M, Tsai AG (2005) Increase plasma viscosity sustains microcirculation after resuscitation from hemorrhagic shock and continuous bleeding. Shock 23:549-555

22. Kretschmar K, Engelhardt T (1994) Swelling of capillary endothelial cells contributes to traumatic hemorrhagic shock-induced microvascular injury: a morphologic and morphometric analysis. Int J Microcirc Clin Exp 14:45-49

23. Pruefer D, Makowski J, Dahm M et al (2003) Aprotinin inhibits leukocyte-endothelial cell interactions after hemorrhage and reperfusion. Ann Thorac Surg 75:210-5, discussion 215-6

24. Nemeth N, Furka I, Miko I (2014) Hemorheological changes in ischemia-reperfusion: an overview on our experimental surgical data. Clin Hemorheol Microcirc 57:215-225. doi:10.3233/CH-131648

25. Wu C-Y, Chan K-C, Cheng Y-J et al (2015) Effects of different types of fluid resuscitation for hemorrhagic shock on splanchnic organ microcirculation and renal reactive oxygen species formation. Crit Care 19:1-13. doi:10.1186/s13054-015-1135-y

26. Zhao L, Wang B, You G et al (2009) Effects of different resuscitation fluids on the rheologic behavior of red blood cells, blood viscosity and plasma viscosity in experimental hemorrhagic shock. Resuscitation 80:253-258. doi:10.1016/j.resuscitation.2008.10.014

27. Guerci P, Tran N, Menu P et al (2014) Impact of fluid resuscitation with hypertonic-hydroxyethyl starch versus lactated ringer on hemorheology and microcirculation in hemorrhagic shock. Clin Hemorheol Microcirc 56:301-317. doi:10.3233/CH-141663

28. Maier S, Holz-Hölzl C, Pajk W et al (2009) Microcirculatory parameters after isotonic and hypertonic colloidal fluid resuscitation in acute hemorrhagic shock. J Trauma 66:337-345. doi:10.1097/TA.0b013e31817dac66

29. Ospina-Tascón GA, Hernandez G, Cecconi M (2016) Understanding the venous-arterial CO2 to arterial-venous O2 content difference ratio. Intensive Care Med 1-4. doi: 10.1007/s00134-016-4233-7

\section{Submit your manuscript to a SpringerOpen ${ }^{\circ}$ journal and benefit from:}

- Convenient online submission

- Rigorous peer review

- Immediate publication on acceptance

- Open access: articles freely available online

- High visibility within the field

- Retaining the copyright to your article

Submit your next manuscript at $>$ springeropen.com 